\title{
On the zero forcing number and spectral radius of graphs
}

\author{
Wenqian Zhang \\ School of Mathematics and Statistics \\ Shandong University of Technology \\ Zibo 255049, China \\ zhangwq@sdut.edu.cn \\ Weifan Wang ${ }^{\ddagger}$ \\ Department of Mathematics \\ Zhejiang Normal University \\ Jinhua 321004, China \\ wwf@zjnu.cn
}

\author{
Jianfeng Wang* $\dagger$ \\ School of Mathematics and Statistics \\ Shandong University of Technology \\ Zibo 255049, China \\ jfwang@sdut.edu.cn
}

\section{Shengjin Ji}

School of Mathematics and Statistics

Shandong University of Technology

Zibo 255049, China

jishengjin20130163.com

Submitted: Aug 13, 2021; Accepted: Feb 6, 2022; Published: Feb 25, 2022

(C) The authors. Released under the CC BY-ND license (International 4.0).

\begin{abstract}
In this paper, we determine the graphs (respectively, trees) with maximum spectral radius among all graphs (respectively, trees) with zero forcing number at most $k$. As an application, we give a sharp lower bound for the zero forcing number of graphs involving the spectral radius.
\end{abstract}

Mathematics Subject Classifications: 05C50, 05C15

\section{Introduction}

All graphs consider here are simple, finite and undirected. For any notation and terminology used but not defined, one may refer to $[9,15]$. Let $G=(V(G), E(G))$ be such a graph with order $n=|V(G)|$. The color-change rule on $G$ is defined as follows. Originally, color the vertices in $V(G)$ and its subset $S$ to be white and blue respectively. For each of the

${ }^{*}$ Corresponding author.

${ }^{\dagger}$ Supported by NSFC grant 11971274 .

${ }^{\ddagger}$ Supported by NSFC grant 11771402 . 
steps, a blue vertex $u$ in $S$ with exactly one white neighbor $v$ will force $v$ to turn into blue. At this time, we say $u$ forces $v$. The set $S$ is said to be a zero forcing set if all vertices of $V(G)$ become blue by applying the color-change rule. And this process is called the forcing process of $S$ in $G$. The zero forcing number of $G$ is the minimum cardinality of zero forcing sets of $G$, denoted by $Z(G)$.

As pointed in $[20,26]$, the forcing process is an instance of a propagation process on graphs, and it has many applications to other branches of mathematics, computer science and physics, such as the linear and quantum controllability for systems that stem from networks $[11,12]$ and the power domination [34]. Moreover, diverse graph processes are employed to model technical or societal processes in other fields. For more details and an overview of the different models and applications, refer to the book [5] and the papers $[20,26]$ with the references therein.

Notice that the zero forcing process was proposed in [13]. Independently, the zero forcing number was introduced in [1] as a bound for the minimum rank or, equivalently, the maximum nullity $M(G)$ of a graph $G$. Recall, the minimum rank of $G$ is defined to be the smallest possible rank over all symmetric real matrices whose $i j$-th entry (for $i \neq j)$ is nonzero whenever $i j \in E(G)$ in $G$ and is zero otherwise. Then, the minimum rank of $G$ is $n-M(G)$. In [2], it was shown that $Z(G) \geqslant M(G)$ for any graph $G$. This parameter has been extensively studied in over half a century, largely due to its connection to inverse eigenvalue problems for graphs, singular graphs, biclique partitions, as well as its applications to other problems and disciplines. For more results on this topic, refer to $[1,22,35]$.

Recently, it has attracted great concern about the relations between the zero forcing number and other graph parameters, such as the connected domination number [3], the tree-width [4], the degree sequence [14, 24], the perfect dominating set [19], the path cover number [23], the girth and minimum degree [26], the Grundy (total) domination number $[8,29]$ and the chromatic number [32]. Up to now, a mass of papers about the zero forcing number have been published. We here tend not to list all, but we focus primarily on those related to the lower bounds for the zero forcing number. Let $\delta$ and $g$ be the minimum degree and the girth of a graph $G$. Recall that the girth is the length of the shortest cycle in a graph. Easily to see that a trivial but tight one is $Z(G) \geqslant \delta$. Whereas, if $g \geqslant 3$ and $\delta \geqslant 2$, Davila, Kalinowski, and Stephen [17] showed that $Z(G) \geqslant \delta+(\delta-2)(g-3)$, confirming the earlier conjecture from [18]. Kalinowski, Kamčev and Sudakov [26] essentially improved this bound with few exception of very small values of $\delta$ and $g=3$, 4 , whose bound is stated as follows:

$$
Z(G) \geqslant \begin{cases}e^{-1}\left(\frac{\delta^{k}}{k+1}-\delta^{k-1}\right) & \text { if } g=2 k+1 \\ 2 e^{-1}\left(\frac{\delta^{k}}{k+1}-\delta^{k-1}\right) & \text { if } g=2 k+1\end{cases}
$$

Further, Inq. (1) was extended to $H$-free graphs by their method based on the Turán numbers [26]. When it comes to our interests, we prefer to their spectral bounds on the zero forcing number of a graph. Some jargon from spectral graph theory are now introduced. The adjacency matrix $A(G)=\left(a_{i j}\right)$ of a graph $G$ with order $n$ is an $n \times n$ 


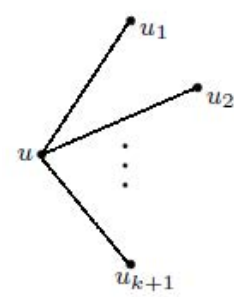

$n=k+2$

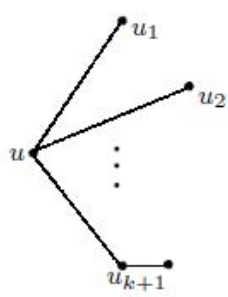

$n=k+3$

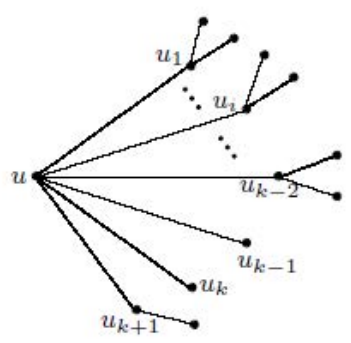

$k+4 \leq n \leq 3 k-1$

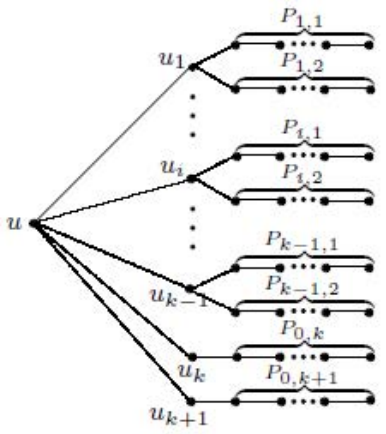

$n \geq 3 k$

Figure 1: The tree $T_{n, k}$.

matrix whose entry $a_{i j}$ is 1 if $i j \in E(G)$, and 0 otherwise. The eigenvalues of $G$ are those of its adjacency matrix. An $(n, d, \lambda)$-graph is a $d$-regular graph with order $n$ in which all eigenvalues but the largest one are at most $\lambda$ in absolute value. Using the well-known estimate on the edge distribution of a graph in terms of its eigenvalues (eg, [27]), Kalinowski, Kamčev and Sudakov [26] provided the following spectral bounds for the $(n, d, \lambda)$-graphs $G$ :

$$
Z(G) \geqslant n\left(1+\frac{2 \lambda_{\min }}{d-\lambda_{\min }}\right),
$$

where $\lambda_{\min }$ is the smallest eigenvalue of $G$, and the bound is tight. Remember that the spectral radius, denoted by $\rho(G)=\lambda_{\max }$, of $G$ is its largest eigenvalue. In the paper, we will investigate the relations between the zero forcing number and the spectral radius of a graph.

Let $\mathscr{S}_{n, k}$ be the set of all graphs with order $n$ and zero forcing number at most $k$ $(1 \leqslant k \leqslant n-1)$. The first goal of this paper is about to determine the extremal graphs with maximum spectral radius in $\mathscr{S}_{n, k}$. This is the type of Brualdi-Solheid Problem [10] that determining the maximal spectral radius of graphs in a given family, with a considerable number of results having been published (see [7, 16, 21] for examples).

Let $G \nabla H$ be the join obtained from two disjoint graphs $G$ and $H$ by connecting each vertex of $G$ to each vertex of $H$. As usual, let $K_{n}$ and $P_{n}$ be the complete graph and the path with order $n$ respectively. It is the time to state the first main result in the paper.

Theorem 1. In $\mathscr{S}_{n, k}$, the maximum spectral radius is attained by the following unique graph:

(i) $P_{n}$ if $k=1$;

(ii) $G_{n, k}=K_{k-1} \nabla P_{n-k+1}$ if $2 \leqslant k \leqslant n-2$;

(iii) $K_{n}$ if $k=n-1$.

We next consider the same problem for the trees. Let $\mathscr{T}_{n, k}$ be the set of all trees with order $n$ and zero forcing number at most $k$, where $k \geqslant 1$ and $n \geqslant k+2$. 
Theorem 2. In $\mathscr{T}_{n, k}$, the maximum spectral radius is attained by the following unique tree:

(i) $P_{n}$ if $k=1$;

(ii) $T_{n, k}$ if $2 \leqslant k \leqslant n-2$,

where $T_{n, k}$ is shown in Fig. 1 and minutely defined in Definition 13.

Note, in the tree $T_{n, k}$ with $n \geqslant 3 k$, that the pendant paths attached at the vertices $u_{i}$ 's $(1 \leqslant i \leqslant k+1)$ are of almost equal length, and $\min \left\{\left|P_{i, 1}\right|,\left|P_{i, 2}\right|\right\} \geqslant \max \left\{\left|P_{0, k}\right|,\left|P_{0, k-1}\right|\right\}$ $(1 \leqslant i \leqslant k-1)$ as well as $\min \left\{\left|P_{i, 1}\right|,\left|P_{i, 2}\right|\right\} \geqslant \max \left\{\left|P_{j, 1}\right|,\left|P_{j, 2}\right|\right\}(1 \leqslant i<j \leqslant k-1)$, where $\left|P_{i, j}\right|$ means the length of pendant path $P_{i, j}$.

In the end, on the basis of Theorem 1, we can provide the following lower bound for the zero forcing number of a graph by means of its spectral radius.

Theorem 3. Let $G$ be a graph with order $n$ and spectral radius $\lambda_{\max }$. Then

$$
Z(G) \geqslant 1+\left\lfloor\frac{n+\lambda_{\max }-\sqrt{n^{2}+2\left(\lambda_{\max }-2\right) n-3 \lambda_{\max }^{2}+12}}{2}\right\rfloor .
$$

Moreover, this bound is sharp.

Here is the remainder of the paper. In Section 2 we give a proof of Theorem 1 by employing the $k$-trees. In Section 3, to show Theorem 2, we mainly use several old and new grafting transformations on the spectral radius of graphs. In Section 4, we provide a proof of Theorem 3, which is based on Theorem 1 and some spectral techniques including the equitable partition.

\section{Proof of Theorem 1}

The following lemma follows from the well-know Perron-Frobenius Theorem, referring to Theorem 2.2.1 in [9].

Lemma 4. Let $G$ be a connected graph, and $H$ be a subgraph of $G$. Then $\rho(H) \leqslant \rho(G)$, and equality holds if and only if $H=G$.

A column vector $\mathbf{x}$ indexed by the vertices of $G$ can be denoted by $\mathbf{x}=\left(x_{1}, x_{2}, \ldots, x_{n}\right)^{t}$, where $x_{i}$ is the component of $\mathbf{x}$ corresponding to the vertex $i$ for any $1 \leqslant i \leqslant n$, and the sign $t$ means the transpose of the vector. If $G$ is connected, from Perron-Frobenius Theorem it follows that $\rho(G)$ is simple and its associated eigenvector, named as Perron vector, is positive.

The following lemma is a generalization of Theorem 8.1.3 in [15].

Lemma 5. Let $G$ be a connected graph with Perron vector $\mathbf{x}=\left(x_{1}, x_{2}, \ldots, x_{n}\right)^{t}$. Let $u, v$, be any two vertices, and $W$ be a set of vertices which are adjacent to $u$ but not adjacent to $v$. Let $G^{\prime}$ be the graph obtained from $G$ by deleting the edges between $u$ and $W$, and adding the edges between $v$ and $W$. If $x_{v} \geqslant x_{u}$, then $\rho\left(G^{\prime}\right)>\rho(G)$. 
For an integer $r \geqslant 1$, a subset $V^{\prime} \subseteq V(G)$ is called a $r$-clique of a graph $G$ if $G\left[V^{\prime}\right]$, the subgraph of $G$ induced by $V^{\prime}$, is a compete graph on $r$ vertices. A $k$-tree is a chordal graph which has an inductive definition that extends the definition of a tree, which was introduced by Beineke and Pippert in [6].

Definition 6. [6] For an integer $k \geqslant 1$, a $k$-tree can be inductively defined as follows:

(i) Every complete graph with $k+1$ vertices is a $k$-tree;

(ii) If $G$ is a $k$-tree, $v \notin V(G)$ and $V^{\prime} \subseteq V(G)$ induces a $k$-clique of $G$, then $G^{\prime}=$ $\left(V(G) \cup\{v\}, E(G) \cup(v, w) \mid w \in V^{\prime}\right)$ is also a $k$-tree;

(iii) Nothing else is a $k$-tree.

Let $\mathscr{K}_{n, k}$ be the family of all the $k$-trees with order $n$ and zero forcing number $k$. Let $G$ be a graph on $n$ vertices with $Z(G)=k$. Clearly, $k=1$ iff $G=P_{n}$. If $k=n-1$, then $\rho(G) \leqslant \rho\left(K_{n}\right)$ and equality holds iff $G=K_{n}$. In what follows we set $2 \leqslant k \leqslant n-2$.

Mitchell [30] showed the following useful result.

Proposition 7. [30] Let $G$ be a graph in $\mathscr{S}_{n, k}$ with $2 \leqslant k \leqslant n-2$. Then $G$ is a spanning subgraph of some graph in $\mathscr{K}_{n, k}$.

Note, any $k$-tree with order $n$ has precisely $\frac{k(k-1)}{2}+k(n-k)$ edges. For any graph $G \in \mathscr{K}_{n, k}$, every vertex has degree at least $k$. Since $Z(G)=k$, for any minimum zero forcing set $Z \subseteq V(G)$, the first forcing vertex must have degree $k$, and its neighbourhood must induce a $k$-clique (since each vertex of a $k$-tree is contained in a $(k+1)$-clique). Recall, a vertex $u \in V(G)$ is called a leaf if its degree $d(u)=1$.

Proposition 8. Let $2 \leqslant k \leqslant n-2$. Then the graph $G_{n, k}=K_{k-1} \nabla P_{n-k+1}$ is the unique graph maximizing the spectral radius in $\mathscr{K}_{n, k}$.

Proof: Clearly, $G_{n, k}=K_{k-1} \nabla P_{n-k+1}$ is a $k$-tree with zero forcing number $k$, since the set of vertices in $K_{k-1}$ and a leaf of $P_{n-k+1}$ is a zero forcing set with $k$ vertices.

Let $G$ be a $k$-tree in $\mathscr{K}_{n, k}$ with maximum spectral radius. Set $\mathbf{x}=\left(x_{1}, x_{2}, \ldots, x_{n}\right)^{t}$ to be its Perron vector. Due to $Z(G)=k$, suppose that $Z=\left\{u_{1}, u_{2}, \ldots, u_{k}\right\}$ is a zero forcing set of $G$. As mentioned above, $G[Z]$, the induced subgraph by $Z$, is a $k$-clique of $G$. Let the vertices of $Z$ be initially blue in $G$. Then by color-change rule, without loss of generality, we can denote the other vertices (not in $Z$ ) of $G$ which are forced blue in order by $u_{k+1}, u_{k+2}, \ldots, u_{n}$. In detail, let the zero forcing process starting with $Z$ be described as $v_{1} \rightarrow u_{k+1}, v_{2} \rightarrow u_{k+2}, \ldots, v_{n-k} \rightarrow u_{n}$, where $v \rightarrow u$ indicates that vertex $v$ forces vertex $u$.

Notice that for any $i<j$, if $u_{i}$ forces $u_{j}$, by color-change rule we know that $u_{i}$ is adjacent to $u_{j}$ but not adjacent to any vertex $u_{\ell}$ for $\ell \geqslant j+1$. For any $t \geqslant k$, set $Z_{t}=\left\{u_{1}, u_{2}, \ldots, u_{t}\right\}$. For any vertex $u$ of $G$, let $N_{G}(u)$ denote the neighbourhood of $u$. Then the following claims are easy to establish:

(i) $G\left[Z_{k+1}\right]$ is a $(k+1)$-clique, and $N_{G}\left(v_{1}\right)=Z_{k+1}-\left\{v_{1}\right\}$; 
(ii) $N_{G}\left(v_{j}\right) \bigcap\left\{u_{k+j}, u_{k+j+1}, \ldots, u_{n}\right\}=\left\{u_{k+j}\right\}$ for all $j \in\{1,2, \ldots, n-k\}$;

(iii) $N_{G}\left(u_{j}\right) \bigcap Z_{j-1}=Z_{j-1}-\left\{v_{1}, v_{2}, \ldots, v_{j-1-k}\right\}$ for all $j \in\{k+2, k+3, \ldots, n\}$.

Notice that for $k+1 \leqslant j \leqslant n$, the vertex $u_{j}$ has precisely $k$ neighbors in $Z_{j-1}$. Moreover, for any $k+1 \leqslant j \leqslant n-1, u_{j}$ is adjacent to the vertex $u_{j+1}$, and $v_{j-k}$ is the unique vertex in $Z_{j-1}$ which is adjacent to $u_{j}$ but not adjacent to $u_{j+1}$ (This fact will be used repeatedly in the following paragraph, which is denoted by Fact*).

Let $Z^{\prime}=V(G)-\left(\left\{v_{1}, v_{2}, \ldots, v_{n-1-k}\right\} \cup\left\{u_{n-1}\right\}\right)$. We shall prove $Z^{\prime}$ is another zero forcing set of $G$ with $k$ vertices, and the forcing process starting from $Z^{\prime}$ is $u_{n} \rightarrow u_{n-1}, u_{n-1} \rightarrow$ $v_{n-1-k}, u_{n-2} \rightarrow v_{n-2-k}, \ldots, u_{k+1} \rightarrow v_{1}$ (Note, $Z^{\prime}$ is actually the reversal of $Z$, which is also a zero forcing set by [2]. But for the completeness of the proof, we give the details here). Clearly, $G\left[Z^{\prime}\right]$ is a $k$-clique containing $u_{n}$ as a vertex. Now color the vertices in $Z^{\prime}$ to be blue initially. By letting $j=n$ in (iii), we have that $u_{n-1}$ is the unique neighbor with white color of $u_{n}$. Then by the color-change rule, the vertex $u_{n}$ forces the vertex $u_{n-1}$. Hence, the vertex $u_{n}$ and its neighbors are all forced blue. By Fact*, $v_{n-1-k}$ is the unique vertex except the neighbors of $u_{n}$, which is adjacent to $u_{n-1}$. Then, $u_{n-1}$ forces $v_{n-1-k}$, and hence the vertex $u_{n-1}$ and its neighbors are all forced blue. Again by Fact*, $v_{n-2-k}$ is the unique vertex except the neighbors of $u_{n-1}$, which is adjacent to $u_{n-2}$. Then, $u_{n-2}$ forces $v_{n-2-k}$, and hence the vertex $u_{n-2}$ and its neighbors are all forced blue. Continue the process until the vertex $u_{k+1}$ and its neighbors are all forced blue. Consequently, all the vertices of $G$ are forced blue. Therefore, $Z^{\prime}$ is a zero forcing set of $G$.

Let $p_{1}, p_{2}, \ldots, p_{k-1}$ be $k-1$ vertices of $G$ such that their corresponding components $x_{p_{1}}, \ldots, x_{p_{k-1}}$ in $\mathbf{x}$ are the first $k-1$ largest ones among all the vertices of $G$. For $1 \leqslant i \leqslant k-1$, we shall prove $d_{G}\left(p_{i}\right)=n-1$, where $d_{G}\left(p_{i}\right)$ is the degree of vertex $p_{i}$ in $G$. Assume by contradiction that $d_{G}\left(p_{j}\right) \leqslant n-2$ for some $1 \leqslant j \leqslant k-1$. We next distinguish into the following two cases.

Case 1. The vertex $p_{j} \in Z \bigcup\left\{u_{k+1}\right\}$. In the forcing process starting from $Z$, let $u_{\ell}$ be the first vertex which is not adjacent to vertex $p_{j}$, where $\ell \geqslant k+2$. (Then we must have $p_{j} \rightarrow u_{\ell-1}$.) Since the vertex $u_{\ell}$ has exactly $k$ neighbors in $Z_{\ell-1}$, then there is a neighbor (say, $u_{b}$ ) of $u_{\ell}$ in $Z_{\ell-1}$ such that $u_{b} \notin\left\{u_{\ell-1}, p_{1}, p_{2}, \ldots, p_{k-1}\right\}$, and thus $x_{u_{b}} \leqslant x_{p_{j}}$. For $\ell \leqslant i \leqslant n$, if $u_{i}$ is adjacent to $u_{b}$ (notice that $u_{i}$ is not adjacent to $p_{j}$ ), after deleting the edge $u_{b} u_{i}$ and adding the edge $p_{j} u_{i}$ to $G$, we obtain the graph $G_{1}$. For any $\ell \leqslant i \leqslant n$, since $p_{j}$ is adjacent to all other vertices in $G_{1}\left[Z_{i-1}\right]$, then the neighbors within $Z_{i-1}$ of $u_{i}$ in $G_{1}$ induces a $k$-clique of $G_{1}\left[Z_{i-1}\right]$. Thus $G_{1}$ is a $k$-tree on $n$ vertices. Furthermore, $Z$ is also a zero forcing set in $G_{1}$ (we just change $p_{j} \rightarrow u_{\ell-1}$ to $u_{b} \rightarrow u_{\ell-1}$ in the forcing process of $Z$, and change $u_{b} \rightarrow v$ to $p_{j} \rightarrow v$ in $G_{1}$ if $u_{b} \rightarrow v$ in $G$ for some vertex $v$ ). Hence $G_{1}$ is a $k$-tree on $n$ vertices with zero forcing number $Z\left(G_{1}\right)=k$. Thereby, $G_{1} \in \mathscr{K}_{n, k}$. By Lemma 5 we get $\rho(G)<\rho\left(G_{1}\right)$, a contradiction.

Case 2. The vertex $p_{j} \notin Z \bigcup\left\{u_{k+1}\right\}$. Then we have $p_{j}=u_{m}$ for some $k+2 \leqslant m \leqslant n$. In the forcing process starting from $Z$, if there exists a vertex $u_{i}(i \geqslant m+1)$ which is not adjacent to $u_{m}$, then we can assume that $u_{\ell}$ is such a vertex with $\ell(\geqslant m+1)$ being minimum. Since the vertex $u_{\ell}$ has precisely $k$ neighbors in $Z_{\ell-1}$, there is a neighbor (say, $u_{c}$ ) of $u_{\ell}$ in $Z_{\ell-1}$ such that $u_{c} \notin\left\{u_{\ell-1}, p_{1}, p_{2}, \ldots, p_{k-1}\right\}$, and thus $x_{u_{c}} \leqslant x_{u_{m}}$. For $\ell \leqslant i \leqslant n$, 
if $u_{i}$ is adjacent to $u_{c}$ (notice that $u_{i}$ is not adjacent to $u_{m}$ ), by deleting the edge $u_{c} u_{i}$ and adding the edge $u_{m} u_{i}$ in $G$, we obtain the graph $G_{2}$. But $G_{2}$ is a $k$-tree on $n$ vertices with zero forcing number $Z\left(G_{2}\right)=k$, with a similar discussion in Case 1 . Hence, $G_{2} \in \mathscr{K}_{n, k}$. From Lemma 5 it follows that $\rho(G)<\rho\left(G_{2}\right)$, a contradiction again.

Consequently, we have that, for any $i \geqslant m+1$, the vertex $u_{i}$ is adjacent to the vertex $u_{m}$. In particular, $u_{m}$ is adjacent to $u_{n}$. Since $d_{G}\left(u_{n}\right)=k$, thus $u_{m}$ is contained in the (unique) $(k+1)$-clique containing $u_{n}$ in $G$. Recall, $u_{m}=p_{j}$. Then $p_{j} \in Z^{\prime} \bigcup\left\{u_{n-1}\right\}$. Considering the forcing process starting from $Z^{\prime}$, we will have a contradiction by a similar way to Case 1.

As proved above, $d_{G}\left(p_{i}\right)=n-1$ for any $1 \leqslant i \leqslant k-1$. Then, for $1 \leqslant i \leqslant k-1, p_{i}$ must be in $Z \cup\left\{u_{k+1}\right\}$, and thus $G\left[V(G) \backslash\left\{p_{1}, p_{2}, \ldots, p_{k-1}\right\}\right]$ is a tree on $n+1-k$ vertices with zero forcing number 1 , and thus $G\left[V(G) \backslash\left\{p_{1}, p_{2}, \ldots, p_{k-1}\right\}\right]$ is the path on $n+1-k$ vertices. As a consequence, $G$ is the graph $G_{n, k}=K_{k-1} \nabla P_{n+1-k}$, as required.

This completes the proof.

Proof of Theorem 1: Let $G$ be a graph $G$ in $\mathscr{S}_{n, k}$ with $2 \leqslant k \leqslant n-2$. By Proposition 7 there exists a graph $H \in \mathscr{K}_{n, k}$ such that $G$ is a spanning subgraph of $H$. From Lemma 4 and Proposition 8, it follows that $\rho(G) \leqslant \rho(H) \leqslant \rho\left(G_{n, k}\right)$, where $\rho(G)=\rho\left(G_{n, k}\right)$ iff $G=G_{n, k}$.

This ends the proof.

\section{Proof of Theorem 3}

The study of spectral radius and its relation to other graph parameters is a hot area of research with many different techniques and applications, surveyed in the monographs of, eg., $[9,15,25]$. Due to Theorem 1, we are now in the stage to show Theorem 3.

Proof of Theorem 3: If $G$ is edgeless, then $\rho(G)=0$, and thus the inequality (3) holds as $Z(G) \geqslant 1$. Since $Z\left(P_{n}\right)=1$ and $\rho\left(P_{n}\right)<2$, the inequality (3) holds for $G=P_{n}$. We next assume that $G \neq P_{n}$ contains at least one edge. Let $Z(G)=k$. Then $k \geqslant 2$ and $1 \leqslant \rho(G) \leqslant n-1$.

For convenience, set $\rho(G)=\lambda_{\max }=\rho$. We prove Ineq. (3) by contradiction. Assume that

$$
k=Z(G) \leqslant\left\lfloor\frac{1}{2}\left(n+\rho-\sqrt{n^{2}+2(\rho-2) n-3 \rho^{2}+12}\right)\right\rfloor .
$$

Due to $\rho \geqslant 1$, a direct calculation shows that

$$
\frac{1}{2}\left(n+\rho-\sqrt{n^{2}+2(\rho-2) n-3 \rho^{2}+12}\right) \leqslant 2 \rho,
$$

which together with $(4)$ results in $2 \rho-k \geqslant 0$. On the other hand, it is routine to verify

$$
\left\lfloor\frac{1}{2}\left(n+\rho-\sqrt{n^{2}+2(\rho-2) n-3 \rho^{2}+12}\right)\right\rfloor \leqslant n-2 .
$$


Hence $2 \leqslant k \leqslant n-2$. By Theorem 1 we get $\rho \leqslant \rho\left(G_{n, k}\right)$. From Lemma 4 it follows that $\rho\left(G_{n, k}\right)<\rho\left(K_{k-1} \nabla C_{n+1-k}\right)$, where $C_{n+1-k}$ is the cycle with order $n+1-k$. By the obvious equitable partition of the graph $K_{k-1} \nabla C_{n+1-k}$ (see [9, Chapter 2], or [15, Theorem 2.1.8]), it is easy to obtain that

$$
\rho\left(K_{k-1} \nabla C_{n+1-k}\right)=\frac{k+\sqrt{4(k-1) n-3 k^{2}+12}}{2} .
$$

Then

$$
\rho<\frac{k+\sqrt{4(k-1) n-3 k^{2}+12}}{2} \text {, and thus } 2 \rho-k<\sqrt{4(k-1) n-3 k^{2}+12} .
$$

Squaring both sides of the above inequality, we get $4 k^{2}-4 k(\rho+n)+4\left(\rho^{2}+n-3\right)<0$. Hence,

$$
k>\frac{1}{2}\left(n+\rho-\sqrt{n^{2}+2(\rho-2) n-3 \rho^{2}+12}\right),
$$

which is contradictive to the assumption (4).

We next show that this bound is sharp. Note that $\rho\left(P_{n}\right)=2 \cos \frac{\pi}{n+1}$ and $Z\left(P_{n}\right)=1$ and $\rho\left(K_{n}\right)=Z\left(K_{n}\right)=n-1$. Easily to verify that $P_{n}$ and $K_{n}$ satisfy the equality in Theorem 3 .

In fact, the graph $G_{n, k}$ also satisfies the equality in Theorem 3. Otherwise, from Theorem 3 it follows that

$$
Z\left(G_{n, k}\right)=k \geqslant 2+\left\lfloor\frac{1}{2}\left(n+\rho\left(G_{n, k}\right)-\sqrt{n^{2}+2\left(\rho\left(G_{n, k}\right)-2\right) n-3 \rho^{2}\left(G_{n, k}\right)+12}\right)\right\rfloor,
$$

which results in

$$
k-1>\frac{1}{2}\left(n+\rho\left(G_{n, k}\right)-\sqrt{n^{2}+2\left(\rho\left(G_{n, k}\right)-2\right) n-3 \rho^{2}\left(G_{n, k}\right)+12}\right) .
$$

Since $G_{n, k}$ contains $K_{k+1}$ as a subgraph, then $\rho\left(G_{n, k}\right) \geqslant k$. Along with the above inequality we obtain

$$
\rho\left(G_{n, k}\right)<\frac{k-1+\sqrt{4(k-2) n-3(k-1)^{2}+12}}{2} .
$$

Note $Z\left(G_{n, k}\right)=k$. As proved above, $\rho(H)=\frac{k+\sqrt{4(k-1) n-3 k^{2}+12}}{2}$ with $H=K_{k-1} \nabla C_{n+1-k}$. Let $\mathbf{x}=\left(x_{i}\right)$ be the Perron vector of $H$ corresponding to $\rho(H)$. By the symmetry, we can set $x_{u}=a$ if $u$ is in $V\left(K_{k-1}\right)$, and $x_{u}=b$ if $u$ is in $V\left(C_{n+1-k}\right)$. Hence, $(k-1) a^{2}+(n+$ $1-k) b^{2}=1$. Clearly, $a \geqslant b$, and thus $b^{2} \leqslant \frac{1}{n}$. Then

$$
\rho\left(G_{n, k}\right) \geqslant \mathbf{x}^{\mathbf{t}} A\left(G_{n, k}\right) \mathbf{x}=\rho(H)-2 b^{2} \geqslant \rho(H)-\frac{2}{n} .
$$

Combining (5) and (6) we get

$$
\frac{k+\sqrt{4(k-1) n-3 k^{2}+12}}{2}-\frac{2}{n}<\frac{k-1+\sqrt{4(k-2) n-3(k-1)^{2}+12}}{2},
$$


which is equivalent to

$$
1-\frac{4}{n}<\frac{6 k-4 n-3}{\sqrt{4(k-1) n-3 k^{2}+12}+\sqrt{4(k-2) n-3(k-1)^{2}+12}} .
$$

Then by $k \geqslant 2$ and $n \geqslant k+2$ we have

$$
1-\frac{4}{k+2}<\frac{2 k-11}{2 k+3}
$$

which yields $3 k+8<0$, a contradiction.

This finishes the proof.

\section{Proof of Theorem 2}

In this section, to show Theorem 2 we firstly introduce two grafting transformations on the spectral radius of graphs, which stem from [28] and can be found in the monograph [15, Theorem 8.1.20] and [15, Theoem 8.1.22].

Lemma 9. [28] Let $G$ be a connected graph on $n \geqslant 2$ vertices, and $v \in V(G)$. Let $G(k, \ell)$ $(k \geqslant \ell \geqslant 1)$ be the graph obtained from $G$ by attaching two pendant paths of length $k$ and $\ell$ at the same vertex $v$. Then $\rho(G(k, \ell))>\rho(G(k+1, \ell-1))$.

Lemma 10. [28] Let $u, v$ be adjacent vertices of a connected graph $G$, both of degree at least 2. Let $G(k, \ell)(k \geqslant \ell \geqslant 1)$ be the graph obtained from $G$ by attaching two pendant paths of length $k$ and $\ell$ at $u$ and $v$ respectively. Then $\rho(G(k, \ell))>\rho(G(k+1, \ell-1))$.

Besides the above two ones, we need the following grafting transformation also.

Lemma 11. Let $G$ be a connected graph and $\mathbf{x}=\left(x_{u}, x_{v}, \cdots, x_{w}\right)^{t}$ be its Perron vector whose components indexed by the vertices of $G$. Suppose $u u_{1} \cdots u_{s}$ and $v v_{1} \cdots v_{t}$ are two pendant paths attached at $u$ and $v$, respectively, where $s>t \geqslant 0$. Let $H$ be the graph obtained from $G$ by deleting edges $u u_{1}$ and $v v_{1}$ (for $t \geqslant 1$ ), and adding edges uv $v_{1}$ and $v u_{1}$. If $x_{v} \geqslant x_{u}$, then $\rho(H)>\rho(G)$.

Proof. We prove the lemma by induction on $t$. For $t=0$ (i.e., $v_{0}=v$ ), the result follows from Lemma 5. Suppose the lemma is true for $t=k-1$, where $k \geqslant 1$. For $t=k$, we prove the lemma by the following two cases.

Case 1. $x_{u_{1}} \leqslant x_{v_{1}}$. Note that $u_{1} u_{2} \cdots u_{s}$ and $v_{1} v_{2} \cdots v_{t}$ are two pendant paths attached at $u_{1}$ and $v_{1}$, respectively. Clearly, $H$ is also the graph obtained from $G$ by deleting edges $u_{1} u_{2}$ and $v_{1} v_{2}$, and adding edges $u_{1} v_{2}$ and $v_{1} u_{2}$. Notice that the length of $v_{1} v_{2} \cdots v_{t}$ is equal to $t-1=k-1$. Along with the induction hypothesis, we have $\rho(H)>\rho(G)$.

Case 2. $x_{u_{1}}>x_{v_{1}}$. From the well-known Rayleigh Theorem it follows that

$$
\begin{aligned}
\rho(H) & \geqslant \mathbf{x}^{\mathbf{t}} A(H) \mathbf{x} \\
& =\mathbf{x}^{\mathbf{t}} A(G) \mathbf{x}+2\left(x_{u} x_{v_{1}}+x_{v} x_{u_{1}}\right)-2\left(x_{u} x_{u_{1}}+x_{v} x_{v_{1}}\right) \\
& =\rho(G)+2\left(x_{u}-x_{v}\right)\left(x_{v_{1}}-x_{u_{1}}\right) \\
& \geqslant \rho(G) .
\end{aligned}
$$


If equality holds, then $x_{u}=x_{v}$, and $\mathbf{x}$ is also the Perron vector of $H$. Hence, $\rho(G) x_{u}-$ $\rho(H) x_{u}=x_{u_{1}}-x_{v_{1}}>0$, and then $\rho(G)>\rho(H)$, a contradiction.

A vertex $u \in V(G)$ is called a major vertex if $d(u) \geqslant 3$, and a branch vertex if it is a major vertex attached $d(u)-1$ pendant paths. $T_{l_{1}, l_{2}, \ldots, l_{k}}$ stands for the starlike tree with a major vertex $u$ of degree $k$ satisfying $T_{l_{1}, l_{2}, \ldots, l_{k}}-u=P_{l_{1}} \cup P_{l_{2}} \cup \cdots \cup P_{l_{k}}$. The following conclusion follows from [33, Lemma 2.6] or [31, Theorem 3.8].

Lemma 12. Let $T$ be a tree on $n$ vertices with at least two major vertices, and $u$ be a branch vertex of $T$. The subtree $T_{1}$ is obtained from $T$ by deleting $u$ and the all vertices in its pendant paths. Then

$$
Z(T)=Z\left(T_{1}\right)+d(u)-2 .
$$

We now introduce the tree $T_{n, k}$ on $n$ vertices with zero forcing number $k$.

Definition 13. Let $n$ and $k$ be two integers with $n \geqslant k+2 \geqslant 4$. Set $K_{1, k+1}$ to be a star with center $u$ and leaves $u_{1}, u_{2}, \ldots, u_{k+1}$. Then $T_{n, k}$ is defined as follows (See Fig. 1):

- For $n \in\{k+2, k+3\}$, let $T_{k+2, k}=K_{1, k+1}$ and $T_{k+3, k}$ be the tree obtained from $K_{1, k+1}$ by attaching a pendant edge at a leaf of $K_{1, k+1}$.

- For $k+4 \leqslant n \leqslant 3 k-1$, let $T_{n, k}$ be the tree obtained from $K_{1, k+1}$ by attaching two pendant edges at the vertex $u_{i}$ for $1 \leqslant i \leqslant\left\lfloor\frac{n-k}{2}\right\rfloor-1$, and attaching $(n-k)-2\left\lfloor\frac{n-k}{2}\right\rfloor$ (equal to 0 or 1 ) pendant edge at the vertex $u_{k+1}$.

- For $n \geqslant 3 k, T_{n, k}$ denotes the tree obtained from $K_{1, k+1}$ by attaching two pendant paths at each $u_{i}$ for $1 \leqslant i \leqslant k-1$ and attaching one pendant path at $u_{j}$ for $j=k, k+1$, such that the pendant paths attached at the vertices $u_{1}, u_{2}, \cdots, u_{k+1}$ are almost equal length; moreover, the lengths of the pendant paths attached at $u_{k}$ and $u_{k+1}$ are not greater than those of pendant paths attached at $u_{1}, u_{2}, \ldots, u_{k-1}$; for any $1 \leqslant i<j \leqslant k-1$, the lengths of the pendant paths attached at $u_{i}$ are not less than those of pendant paths attached at $u_{j}$.

Let $\mathscr{T}_{n, k}^{\prime}$ is the set of all trees with order $n$ and zero forcing number $k$, where $k \geqslant 2$ and $n \geqslant k+2$.

Theorem 14. Let $n$ and $k$ be two integers with $k \geqslant 2$ and $n \geqslant k+2$, and let $T \in \mathscr{T}_{n, k}^{\prime}$. Then $\rho(T) \leqslant \rho\left(T_{n, k}\right)$ with equality if and only if $T \cong T_{n, k}$.

Proof. Suppose that $T \in \mathscr{T}_{n, k}^{\prime}$ has the maximum spectral radius. Clearly, it is sufficient to prove $T \cong T_{n, k}$. If $n \in\{k+2, k+3\}$, by $Z(T)=k$ we arrive at $T \in\left\{T_{k+2, k}, T_{k+3, k}\right\}$ easily.

We next consider $n \geqslant k+4$. Let $\mathbf{x}=\left(x_{1}, x_{2}, \ldots, x_{n}\right)^{t}$ be the Perron vector of $T$. Assume that $T$ has a unique major vertex. Then $T$ is a starlike tree with major vertex $u$ of degree $k+1$, due to $Z(T)=k$. Let $u_{1}, u_{2}, \ldots, u_{k+1}$ be the neighbors of $u$. Without loss of generality, set $x_{u_{1}} \geqslant x_{u_{i}}$ for any $2 \leqslant i \leqslant k+1$. Since $n \geqslant k+4$, there is a leaf, say $v$, which 
is not the neighbor of $u$ or $u_{1}$. Note that $\rho(T)>2$. Then we can deduced that $x_{u_{1}} \geqslant x_{v^{\prime}}$, where $v^{\prime}$ is the unique neighbor of $v$. Let $T_{1}$ be the tree obtained from $T$ by deleting the edge $v v^{\prime}$ and connecting $v$ to $u_{1}$. Using Lemma 12 , we get $Z\left(T_{1}\right)=Z(T)=k$. However, by Lemma 5 we obtain $\rho\left(T_{1}\right)>\rho(T)$, which contradicts the choice of $T$. Thereby, the tree $T$ owns at least two major vertices.

Let $w$ be the vertex of $T$ with $x_{w}=\max \left\{x_{i} \mid 1 \leqslant i \leqslant n\right\}$. By $\rho(T)>2$ we get that $w$ is a major vertex of $T$. Let $w_{1} \neq w$ be any branch vertex of $T$, and $h$ be the (unique) neighbor of $w_{1}$ which does not lie in the pendant paths attached at $w_{1}$. If $h \neq w$, then $x_{h} \leqslant x_{w}$ by the hypothesis. We denote by $T_{2}$ the tree obtained from $T$ by deleting the edge $w_{1} h$ and adding the edge $w_{1} w$. Then by Lemma 12 we deduce that $Z\left(T_{2}\right)=Z(T)=k$. From Lemma 5 it follows that $\rho(T)<\rho\left(T_{2}\right)$, a contradiction. Therefore, $h=w$ and $w_{1}$ is adjacent to $w$. Consequently, each major vertex other than $w$ is a branch vertex of $T$ which is a neighbor of $w$.

Claim 1. The vertex $w$ possesses at least two pendant paths. If not, then $w$ owns $i$ pendant paths $(i=0,1)$. Let $w_{2}$ be a branch vertex of $T$. Construct the tree $T_{3}$ obtained from $T$ by deleting $d\left(w_{2}\right)-1-i$ pendant paths and attaching them to $w$. Using Lemma 12 , we get $Z\left(T_{3}\right)=Z(T)=k$. In terms of Lemma 5 , by $x_{w} \geqslant x_{w_{2}}$ we get $\rho(T)<\rho\left(T_{3}\right)$, a contradiction. Hence, there are at least two pendant paths attached at $w$.

Claim 2. All branch vertices except $w$ of $T$ have degree 3. Otherwise, there exists a branch vertex $w_{3}$ with $d\left(w_{3}\right) \geqslant 4$. Construct a new tree $T_{4}$ obtained from $T$ by grafting one pendant path of $w_{3}$ to $w$. Applying Lemma 12 to $T_{4}$ and $T$, we get $Z\left(T_{4}\right)=Z(T)=k$. Since $x_{w} \geqslant x_{w_{3}}$, by Lemma 5 we have $\rho(T)<\rho\left(T_{4}\right)$, a contradiction.

Using Claims 1 and 2, by Lemma 12 we have $d(w)=k+1$ as $Z(T)=k$. In order to get $T \cong T_{n, k}$, we next distinguish the following two cases.

Case $1 . k+4 \leqslant n \leqslant 3 k-1$. Along with $d(w)=k+1$ we know that $w$ possesses at least three pendant paths.

Claim 3. All the pendant paths attached at $w$ have length one, except possibly one of length two. Assume that there exits a pendant path $P$ of $w$ with length at least three. Construct a new tree $T^{\prime}$ from $T$ by removing the leaf of $P$ and connecting it to the unique neighbor of $w$ in this path $P$. By Lemma 12 , we get $Z\left(T^{\prime}\right)=k$, and so $\rho\left(T^{\prime}\right)>\rho(T)$ by Lemma 5, a contradiction. Suppose that $w$ has two pendant paths $P$ and $P^{\prime}$ with length two. Let $T^{\prime \prime}$ be the tree from $T$ by transferring one leaf in $P$ to the unique neighbor of $w$ in $P^{\prime}$. By Lemmas 12 and 5 we get $Z\left(T^{\prime \prime}\right)=k$ and $\rho(T)<\rho\left(T^{\prime \prime}\right)$, a contradiction.

Claim 4. For any branch vertex $u \neq w$, any pendant path attached at $u$ has length one. In fact, if there is a pendant path $P$ attached at $u$ has length at least two, then let $T_{5}$ be the tree obtained from $T$ by interchanging $P$ with a pendant path of length one attached at $w$. By Lemma 12 we have $Z\left(T_{5}\right)=Z(T)$. But $\rho\left(T_{5}\right)>\rho(T)$ by Lemma 11, a contradiction.

From Claims 2, 3 and 4 it follows that $T \cong T_{n, k}$ defined in Definition 13(ii).

Case 2. $n \geqslant 3 k$. We claim that $w$ possesses exactly two pendant paths. If not, assume that there are at least three pendant paths attached at $w$. Similarly, Claims 3 and 4 are still true. But then $n \leqslant 3 k-1$, contradicting the condition $n \geqslant 3 k$.

Recall $d(w)=k+1$. Label the $k+1$ neighbors of $w$ as $w_{1}, w_{2}, \ldots, w_{k+1}$. Without 
loss of generality, From Claims 2 and the above discussion we can suppose that $d_{w_{i}}=3$ for $1 \leqslant i \leqslant k-1$, and $d_{w_{k+1}} \leqslant d_{w_{k}} \leqslant 2$. By symmetry, we can assume $x_{w_{i}} \geqslant x_{w_{j}}$ for $1 \leqslant i<j \leqslant k-1$. Using the similar methods as Claim 4, by Lemma 11 we get that the length of each pendant path attached at $w$ is not less than that of each pendant path attached at $w_{1}, w_{2}, \ldots, w_{k-1}$, and for any $1 \leqslant i<j \leqslant k-1$ the length of each pendant path attached at $w_{i}$ is not less than that of each pendant path attached at $w_{j}$. Then by Lemmas 9 and 10, we see that all the pendant paths of $T$ are of almost equal length. Hence, $T \cong T_{n, k}$ defined in Definition 13(iii).

Lemma 15. Let $n$ be an integer with $n \geqslant 4$. Then $\rho\left(P_{n}\right)<\rho\left(T_{n, 2}\right)<\rho\left(T_{n, 3}\right)<\cdots<$ $\rho\left(T_{n, n-2}\right)$.

Proof. Let $\mathbf{x}=\left(x_{1}, \cdots, x_{n}\right)$ be the Perron vector of $P_{n}$. Choose $u \in V\left(P_{n}\right)$ with $x_{u}=$ $\max \left\{x_{i} \mid 1 \leqslant i \leqslant n\right\}$. Then $d(u)=2$ by $\rho\left(P_{n}\right)>1$. For $n \geqslant 4$, there exists a leaf, say $v$, which is not adjacent to $u$. Let $T_{1}$ be the tree obtained from $P_{n}$ by removing $v$ and connecting it to $u$. By Lemma 5 we get $\rho\left(P_{n}\right)<\rho\left(T_{1}\right)$. Clearly, $Z\left(T_{1}\right)=2$, and $\rho\left(T_{1}\right) \leqslant \rho\left(T_{n, 2}\right)$ by Theorem 14 .

We now prove $\rho\left(T_{n, k}\right)<\rho\left(T_{n, k+1}\right)$ for $2 \leqslant k \leqslant n-3$. Similarly above, let $\mathbf{x}=\left(x_{i}\right)$ be the Perron vector of $T_{n, k}$ and $u$ be the vertex satisfying $x_{u}=\max \left\{x_{i} \mid 1 \leqslant i \leqslant n\right\}$. By $k \leqslant n-3$, there exists a leaf of $T_{n, k}$, say $v$, which is not adjacent to $u$. Let $T_{2}$ be the tree obtained from $T_{n, k}$ by removing $v$ from one pendant path and connecting to $u$. By Lemma 5 we get $\rho\left(T_{n, k}\right)<\rho\left(T_{2}\right)$. From Lemma 12 it follows that $Z\left(T_{2}\right)=k+1$. In view of Theorem 14, we arrive at $\rho\left(T_{2}\right) \leqslant \rho\left(T_{n, k+1}\right)$, and thus $\rho\left(T_{n, k}\right)<\rho\left(T_{2}\right) \leqslant \rho\left(T_{n, k+1}\right)$. Consequently, $\rho\left(P_{n}\right)<\rho\left(T_{n, 2}\right)<\rho\left(T_{n, 3}\right)<\cdots<\rho\left(T_{n, n-2}\right)$.

This completes the proof.

Proof of Theorem 2: The theorem follows from Theorem 14 and Lemma 15 immediately.

\section{Acknowledgements}

We take this opportunity to thank the anonymous referees for their careful reading of the manuscript and suggestions which have immensely helped us get the article to its present form.

\section{References}

[1] AIM Minimum Rank-Special Graphs Work Group (F. Barioli, W. Barrett, S. Butler, S.M. Cioabă, D. Cvetković, S. M. Fallat, C. Godsil, W. Haemers, L. Hogben, R. Mikkelson, S. Narayan, O. Pryporova, I. Sciriha, W. So, D. Stevanović, H. van der Holst, K. Vander Meulen and A. Wangsness). Zero forcing sets and the minimum rank of graphs. Linear Algebra Appl., 428(7):1628-1648, 2008.

[2] F. Barioli, W. Barrett, S. M. Fallat, H. T. Hall, L. Hogben, B. Shader, P. van den Driessche and $\mathrm{H}$. van der Holst.Zero forcing parameters and minimum rank problems. Linear Algebra Appl., 433(2):401-411, 2010. 
[3] D. Amos, Y. Caro, R. Davila and R. Pepper. Upper bounds on the $k$-forcing number of a graph. Discrete Appl. Math., 181:1-10, 2015.

[4] F. Barioli, W. Barrett, S. Fallat, H.T. Hall, L. Hogben, B. Shader, P. van den Driessche and H. van der Holst. Parameters related to tree-width, zero forcing, and maximum nullity of a graph. J. Graph Theory, 72(2):146-177, 2013.

[5] A. Barrat, M. Barthélemy and A. Vespignani. Dynamical Processes on Complex Networks. Cambridge University Press. Cambridge, 2008.

[6] L. W. Beineke and R. E. Pippert. The number of labeled $k$-dimensional trees. $J$. Comb. Theory, 6(2):200-205, 1969.

[7] A. Berman and X.-D. Zhang. On the spectral radius of graphs with cut vertices. J. Comb. Theory Ser. B, 83(2):233-240, 2001.

[8] B. Brešar, C. Bujtás, T. Gologranc, S. Klavžare, G. Košmrlj, B. Patkós, Z. Tuza and M. Vizer. Grundy dominating sequences and zero forcing sets. Discrete Optim., 26:66-77, 2017.

[9] A. E. Brouwer and W. H. Haemers. Spectra of Graphs. Springer, 2012.

[10] R. A. Brualdi and E. S. Solheid. On the spectral radius complementary acylic matrices of zeros and ones. SIAM J. Algebra Discrete Methods, 7:265-272, 1986.

[11] D. Burgarth, S. Bose, C. Bruder and V. Giovannetti.Local controllability of quantum networks. Phys. Rev. A, 79:060305(R), 2009.

[12] D. Burgarth, D. D’Alessandro, L. Hogben, S. Severini and M. Young. Zero forcing, linear and quantum controllability for systems evolving on networks. IEEE Trans. Automat. Control, 58:2349-2354, 2013.

[13] D. Burgarth and V. Giovannetti. Full control by locally induced relaxation. Phys. Rev. Lett., 99:100501, 2007.

[14] Y. Caro and R. Pepper. Dynamic approach to k-forcing. Theory Appl. Graphs, 2: Art. 2, 2015.

[15] D. Cvetković, P. Rowlinson and S. Simić. An Introduction to the Theory of Graph Spectra. Cambridge, 2010.

[16] E. R. van Dam. Graphs with given diameter maximizing the spectral radius. Linear Algebra Appl., 426(2-3):454-457, 2007.

[17] R. Davila, T. Kalinowski and S. Stephen. A lower bound on the zero forcing number. Discrete Appl. Math., 250:363-367, 2018.

[18] R. Davila and F. Kenter. Bounds for the zero forcing number of graphs with large girth. Theory Appl. Graphs, 2: Art. 1, 2015.

[19] R. Davila and M. A. Henning. Zero forcing versus domination in cubic graphs. J. Combin. Optim., 41:553-577, 2021.

[20] S. English, C. MacRury and P. Prałat. Probabilistic zero forcing on random graphs. European J. Combin., 91:103207, 2021. 
[21] A. Erey. Maximal distance spectral radius of 4-chromatic planar graphs. Linear Algebra Appl., 618:183-202, 2021.

[22] S. M. Fallat and L. Hogben. Minimum rank, maximum nullity, and zero forcing number of graphs, 2nd Edition. In Handbook of Linear Algebra, pages 775-810. CRC Press, 2013.

[23] M. Gentner, L. D. Penso, D. Rautenbach and U. S. Souza. Extremal values and bounds for the zero forcing number. Discrete Appl. Math., 214:196-200, 2016.

[24] M. Gentner and D. Rautenbach. Some bounds on the zero forcing number of a graph. Discrete Appl. Math., 236:203-213, 2018.

[25] C. Godsil and G. Royle. Algebraic Graph Theory. Springer, 2001.

[26] T. Kalinowski, N. Kamčev and B. Sudakov. The zero forcing number of graphs. SIAM J. Discrete Math., 33(1):95-115, 2019.

[27] M. Krivelevich and B. Sudakov. Pseudo-random graphs. In More Sets, Graphs and Numbers, E. Gyori, G. Katona and L. Lovász, eds., Bolyai Soc. Math. Stud. 15, pages 199-262, Springer, 2006.

[28] Q. Li and K. Q. Feng. On the largest eigenvalue of graphs (Chinese). Acta Math. Appl. Sinica, 2:167-175, 1979.

[29] J. C.-H. Lin. Zero forcing number, Grundy domination number, and their variants. Linear Algebra Appl., 563:240-254, 2019.

[30] L. Mitchell. Zero-forcing, treewidth, and graph coloring. Austra. J. Combin., 61(1):19-22, 2015.

[31] D. D. Row. A technique for computing the zero forcing number of a graph with a cut-vertex. Linear Algebra Appl., 436(12):4423-4432, 2012.

[32] F. A. Taklimi. Zero Forcing Sets for Graphs, Ph.D. Thesis. University of Regina, Regina, Canada, 2013.

[33] X. Wang, D. Wong and Y. Zhang. Zero forcing number of a graph in terms of the number of pendant vertices. Linear Multilinear Algebra, 68(7):1424-1433, 2020.

[34] M. Zhao, L. Kang and G. Chang. Power domination in graphs. Discrete Math., 306(15):1812-1816, 2006.

[35] Graph Catalog: Families of Graphs. http://aimath.org/pastworkshops/catalog2.html. 